study was to assess prevalence of osteoporosis in Polish elderly population and to assess risk factors related to osteoporotic fractures

Objectives: The study was based on questionnaires distributed among 645 students ( 61 men, 533 women and 51 not report mean age 69,42 $\pm 5,88$ ) attending the Healthy Aging Academy at Medical University.

Methods: Students were asked to answer 20 questions including information about endured fractures, osteoporosis risk factors, screening densitometry, treatment of osteoporosis, supplementation with vitamin D3 and calcium. Also, questions about classical risk factors for osteoporosis (loss of height $(5 \mathrm{~cm} / \mathrm{year}$ ), family history, falls, loss of weight, treatment with steroids) were included to questionnaire.

Results: Fractures caused by fall from their own height were reported by 180 $(27,91 \%)$. Moreover, 96 (14,88\%) responders without fracture history declared more than $3 \mathrm{~cm}$ decrease of height after their forties, what strongly suggest osteoporotic vertebral fractures. Forty three (24\%) of the respondents had multiple fractures and single fractures were reported by $137(76 \%)$ respondents. The most common localization of fractures was forearm $(57 \%)$, shin bones $(10,4 \%)$ and foot bones (9\%). Among subjects who underwent densitometry after the fracture $32 \%$ had the diagnosis of osteopenia; $19,4 \%$ were diagnosed with osteoporosis and in $23,1 \%$ bone density was within normal limits. The strongest risk factors for osteoporotic fractures were numerous falls per year $(p=0,000)$ and frequent drinking of alcohol $(p=0,008)$. The risk factors of fractures (sum of risk factors for falls and osteoporosis) among people with previous fractures vs. those without fractures were statistically significant $(p=0,037)$. Only $13 \%$ of patient with fracture and $15 \%$ of all patients with osteoporosis and osteopenia were treated by antiosteoporotic drugs (ibandronian/aledronian). Calcium supplementation was received by $30 \%$ of patients with fracture and $43 \%$ supplemented vitamin D3.

Conclusions: The prevalence of osteoporotic fractures in population of relatively well educated population of participants of Third Age University seems to be high. Patients with osteoporosis do not receive appropriate pharmacological prophylactic treatment, pointing at the need for education of primary care physicians and elderly population about burden and consequences of osteoporosis.

Disclosure of Interest: None declared

DOI: 10.1136/annrheumdis-2017-eular.5452

\section{AB1154 CHARACTERIZATION OF CHILEAN PATIENTS WITH PULMONARY DISEASE ASSOCIATED TO A RHEUMATOLOGIC DIAGNOSIS IN AN INTEGRATED POLYCLINIC. HOSPITAL PADRE HURTADO EXPERIENCE}

O. Valenzuela ${ }^{1}$, V. Agar ${ }^{1}$, J.P. Leiva ${ }^{2}$, I. Allende ${ }^{2}$, G. Bozzano $^{2}$, G. Miranda ${ }^{3}$ S.E. Ibáñez Vodnizza ${ }^{1} .{ }^{1}$ Rheumatology department, Clinica Alemana de Santiago/Hospital Padre Hurtado; ${ }^{2}$ Facultad de Medicina Clinica Alemana de Santiago - Universidad del Desarrollo; ${ }^{3}$ Hospital Padre Hurtado, Santiago, RM, Chile

Background: Pulmonary diseases associated to a rheumatologic condition constitute a heterogeneous group of pathologies that are grouped according to their clinical and radiological similarity. There is a perception that the incidence of these diseases has been increasing, however, in Chile, there are no up-to-date publications regarding the characteristics of this group of patients.

Objectives: To describe and characterize patients with pulmonary disease associated to a rheumatologic diagnosis at Hospital Padre Hurtado, Chile, between the years 2014 and 2016.

Methods: Patients with suspected lung involvement associated to a connective tissue disease were evaluated by a bronchopulmonar specialist and a rheumatologist in a joint polyclinic. If the diagnosis was confirmed by the specialist the patient was included in the database. Data collected included age, gender, rheumatologic diagnosis and radiological pattern.

Results: 76 patients were included. $85.5 \%$ were female. The mean age was 58 years. Eighty-nine percent of the patients came from the Rheumatology department. These patients developed respiratory symptoms during the course

Table 1. Rheumatologic diagnosis of 76 patients

\begin{tabular}{lc}
\hline Rheumatoid Arthritis (\%) & $31(40.8)$ \\
Scleroderma (\%) & $8(10.5)$ \\
Inflammatory Myopathy (\%) & $5(6.6)$ \\
Sjögren Syndrome (\%) & $5(6.6)$ \\
Systemic Lupus Erythematosus (\%) & $4(5.3)$ \\
Undifferentiated Connective Tissue Disease (\%) & $3(3.9)$ \\
Mixed Connective Tissue Disease (\%) & $3(3.9)$ \\
Recurrent Polychondritis (\%) & $2(2.6)$ \\
Other diagnosis (\%) & $15(19.7)$ \\
\hline
\end{tabular}

Table 2. Radiological evaluation of 53 patients

Interstitial Lung Disease pattern: Non Usual Interstitial Pneumonia (\%) $26(49.1)$

Interstitial Lung Disease pattern: Usual Interstical Pneumonia (\%) $8(15.1)$

Bronchiolitis (\%)

Emphysema (\%)

Nodules (\%)

Pulmonary embolism (\%)

Bronchiectasis (\%)

Sarcoidosis (\%)

Tracheal stenosis (\%) of their disease. The remaining $11 \%$ were patients controlled in the Pulmonary diseases department, and the respiratory symptoms were the first manifestation of their conective tissue disease.The rheumatologic diagnoses that were concluded in this multidisciplinary polyclinic were distributed as described in Table 1.

$69.7 \%$ of the patients have completed the radiological evaluation (Table 2)

Conclusions: Having a multidisciplinary clinical unit allowed us to order and protocolize the study, follow-up and treatment of the patients with lung involvement associated with a connective tissue disease. Currently, the multidisciplinary management of these patients is indispensable, as described in the literature.

Disclosure of Interest: None declared

DOI: 10.1136/annrheumdis-2017-eular.4109

\section{AB1155 DEPRESSION AND SUICIDALITY ARE COMMON IN PSORIATIC ARTHRITIS AND AXIAL SPONDYLOARTHRITIS, AND RATES ARE COMPARABLE TO THOSE IN PSORIASIS}

A. Sheahan ${ }^{1}$, R. Suruki ${ }^{1}$, P.C. Taylor ${ }^{2}$, V.S. Sloan ${ }^{1} .{ }^{1}$ UCB Pharma, Raleigh, United States; ${ }^{2}$ Kennedy Institute, Oxford, United Kingdom

Background: Depression and suicidality are well-described comorbidities in psoriasis (PSO). The prevalence of these comorbidities in psoriatic arthritis (PsA) and axial spondyloarthritis (axSpA) is less well described.

Objectives: To assess the prevalence of depression and suicidality in PsA and axSpA in the recent literature, and compare rates to $\mathrm{PSO}$.

Methods: For PsA and axSpA, we evaluated the recent English-language literature identified through a PubMed search; we used a recent review and performed a targeted review of the period since the publication in order to establish the rates for PSO for comparison. ${ }^{1}$ Review articles were also examined to identify key publications.

Results: Rates of depression in PSO vary widely, depending on the outcome definition and method of ascertainment. Dowlatshahi et al. reported a pooled rate of $9.0-55.0 \%$, with rates from the literature after the review period ranging from $9.0-39.8 \%$. Rates for suicidality also varied widely, with $2.5-17.3 \%$ of patients (pts) reporting suicidal ideation. The limited data available provide ranges for depression in PsA of $3.4-28.6 \%$, and in axSpA of $3.1-44.0 \%$. The single study that differentiated between ankylosing spondylitis (AS) and non-radiographic (nr)-axSpA did not identify a difference between the two groups. ${ }^{2}$ Very limited data existed on suicidality in PsA and axSpA. For PsA, the incidence rates (IR) of suicidal ideation, attempts, and suicide per 1000 person-years in the UK were $0.4,1.3$, and $<0.001$, respectively ${ }^{3}$ no prevalence data were identified. In a study in China, $2.5 \%$ of pts with axSpA reported a past suicide attempt, ${ }^{4}$ while a study in Turkey found $9.6 \%$ of pts with AS had thoughts of suicide in the past year, but without plans. ${ }^{5}$ Depending on the study, different definitions and tools were used to assess depression (eg. HADS, ICD9, antidepressant use), and even when the same tool was used, different cutoffs for defining depression were implemented (eg. cutoff for HADS ranged from $\geq 5$ to $\geq 11$ ), making comparisons across studies difficult. In a large observational study of AS with matched controls, the IR of depression per 1000 pt-years was 5.48 in AS versus 3.29 without AS, risk ratio $1.63 .^{6}$

Conclusions: Although data are limited, rates of depression and suicidality in PsA and axSpA are comparable to those in PSO. Comparisons between studies and diseases are challenging due to a lack of standardized assessment tools and definitions of depression and suicidality. There are almost no data for nr-axSpA, which unlike AS has no gender predominance. Given that depression in PSO pts is more common in women, ${ }^{7}$ understanding the relative prevalence in AS versus $n r-a x S p A$ would be important. Generating additional data regarding the impact of depression and suicidality in PSA and axSpA should increase awareness among treating physicians.

References:

[1] Dowlatshahi EA. J. Invest Dermatol 2014;134:1542-51.

[2] Klic GE. Medicine (Baltimore) 2014:93:e337.

[3] Hagberg KW. Mod Rheumatol 2016;26:774-9.

[4] Chan CY. Int J Rheum Dis 2014; doi:10.1111/1756-185X.12456.

[5] Saygin C. Clin Exp Rheumatol 2015;33(6 Suppl 94):S30-5.

[6] Shen CC. J Rheumatol 2016;43:625-31.

[7] Lamb RC. Br J Dermatol 2016; doi:10.1111/bjd.14833

Disclosure of Interest: A. Sheahan Employee of: UCB Pharma, R. Suruki Employee of: UCB Pharma, P. Taylor: None declared, V. Sloan Employee of: UCB Pharma

DOI: 10.1136/annrheumdis-2017-eular.2455

\section{AB1156 THE DESCRIPTIVE EPIDEMIOLOGY OF CUTANEOUS LUPUS ERYTHEMATOSUS IN THREE LARGE U.S. ADMINISTRATIVE DATABASES, 2010-2014}

S.A. Hall ${ }^{1}$, L. Li ${ }^{2}$, S. Eaton ${ }^{2}$, C. Musselli ${ }^{2}$, A. Dilley ${ }^{1} .{ }^{1}$ Epidemiology; ${ }^{2}$ Biogen, Cambridge, United States

Background: Previous epidemiologic studies of cutaneous lupus erythematosus (CLE) have not described the pediatric burden, and have focused on specific geographic areas, limiting generalizability.

Objectives: To describe the demographics, comorbid conditions and pharmacy drug utilization of CLE patients, 3 large sources of U.S. administrative claims 